\title{
Adaptor protein Shc acts as an immune-regulator for the LPS-stimulated maturation of bone marrow-derived dendritic cells
}

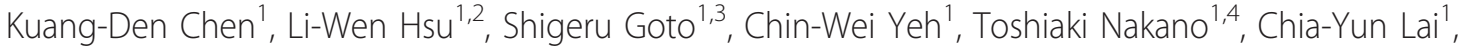 \\ Yen-Chen Chang ${ }^{1}$, Chiung-Hui Hou' ${ }^{1}$, Chih-Chi Wang ${ }^{1}$, Yu-Fan Cheng ${ }^{5}$, King-Wah Chiu ${ }^{6}$, Chih-Che Lin ${ }^{1}$ and \\ Chao-Long Chen ${ }^{1 *}$
}

\begin{abstract}
Background: The Shc isoforms is known to mediate immune responses and has been indicated as a negative regulator of autoimmunity and lymphocyte activation. We aimed to evaluate the immune-regulatory role of Shc in rat bone marrow-derived DCs in the maturation process triggered by LPS.

Results: We found that, in response to LPS, expression of Shc proteins was induced and that neutralization of Shc inhibited the LPS-induced transient phosphorylation of p52Shc on pTyr239/240 in DCs of Lewis (LEW; RT1') rats. Moreover, the significantly enhanced expression of IL-10 and the surface level of costimulatory molecule CD80, as well as suppressed expression of IL- 6 and IL-12 in the Shc-silenced DCs were also observed. Similar I $\kappa B$ phosphorylation occurred in Shc-silenced DCs primed by LPS, indicating Shc is not associated with NF- $\kappa$ B pathway. We further demonstrate that Shc blockade on LPS-treated DCs results in significant increase of the overall STAT3 phosphorylation and the relative levels of phospho-STAT3 in the nuclear fraction. STAT3 activation by LPS with or without Shc blockade was totally abolished by SU6656, a selective Src family kinases inhibitor, underscoring the critical role of Src-mediated activation.

Conclusions: We conclude that Shc blockade in LPS-primed DC leads to the development of tolerogenic DC via Src-dependent STAT3 activation and that adaptor protein Shc might play a pivotal role in mediating immunogenic and tolerogenic properties of DCs.
\end{abstract}

\section{Background}

Dendritic cells (DCs) are the most important APC that play a crucial role in bridging innate resistance and adaptive immunity $[1,2]$. Immature DCs reside in peripheral tissues, where they release soluble mediators (cytokines, chemokines and IFNs) that participate in innate inflammatory responses during infection $[2,3]$. Upon capturing antigens, DCs migrate to the lymph nodes and present processed antigenic epitopes to $\mathrm{T}$ cells, resulting in their activation and further expansion $[4,5]$. A variety of signals induce DC maturation. Mature

\footnotetext{
* Correspondence: clchen@adm.cgmh.org.tw

${ }^{1}$ Center for Translational Research in Biomedical Sciences, Liver

Transplantation Program, Departments of Surgery, Kaohsiung Chang Gung Memorial Hospital and Chang Gung University College of Medicine,

Kaohsiung, Taiwan

Full list of author information is available at the end of the article
}

DCs express high levels of antigen presenting and co-stimulatory molecules and certain cytokines critical for the nature of the $\mathrm{T}$ cell response. For instance, Th1-type $\mathrm{T}$ cell responses need inflammatory IL-12 produced by DCs. Conversely, DCs can also produce anti-inflammatory cytokines, such as IL-10 [6-8], which influences the DC maturation process by down-regulating IL-12 production and thus interfering with the Th1-type $T$ cell responses [4,9-11]. Moreover, IL-10-producing DCs also promote immune tolerance by modulating the suppressive effects of regulatory $\mathrm{T}$ cells $[12,13]$. Accordingly, there has been considerable interest in influencing the $\mathrm{DC}$ maturation process to direct $\mathrm{T}$ cell responses to a desired type (i.e., Th1 vs. Th2/3) for translational purposes.

A useful system for the study of DCs in culture is the use of monocyte-derived DCs obtained in vitro by GM-

\section{Biomed Central}

(c) 2011 Chen et al; licensee BioMed Central Ltd. This is an Open Access article distributed under the terms of the Creative Commons Attribution License (http://creativecommons.org/licenses/by/2.0), which permits unrestricted use, distribution, and reproduction in any medium, provided the original work is properly cited. 
CSF and rIL-4 treatment of peripheral CD14 ${ }^{+}$monocytes. These cells can produce high amounts of cytokines such as IL-6 and IL-12 when stimulated with LPS [14]. Some evidence suggests an involvement of Srcfamily tyrosine kinases (SFKs) in the signaling pathway triggered by LPS. In monocytes, LPS activates the Srcfamily kinase Lyn associated with CD14, a glycosyl phosphatidylinositol (GPI)-anchored molecule that cooperates with toll-like receptor 4 (TLR4) in LPS binding on the surface of these cells [15]. However, the complexity in the engagement of TLRs by LPS leading to interactions with intracellular adaptor proteins and their associated kinases is still under investigation.

Shc adaptor proteins are substrates of receptor tyrosine kinases, and signal events initiated by their phosphorylation culminate in Erk and Jnk activation [16,17]. Among the three related Shc genes, ShcA is ubiquitously expressed, whereas $\mathrm{ShcB}$ and $\mathrm{ShcC}$ are restricted to cells of neural origin, and we describe ShcA here as Shc based on this tissue restriction. Shc is expressed as three isoforms of 46, 52 and $66 \mathrm{kDa}$ derived from ShcA via post-transcriptional splicing, which display the PTB$\mathrm{CH} 1-\mathrm{SH} 2$ Shc family signature, with an added N-terminal $\mathrm{CH} 2$ domain in p66ShcA and a truncated PTB domain in p46ShcA. The PTB and SH2 domains both bind tyrosine-phosphorylated peptides and associate with activated receptor kinases [18]. Recently, it has been found that the defects of pp66ShcA in T cells of $\mathrm{p} 66 \mathrm{ShcA}^{-1-}$ mice display enhanced proliferative responses to $\mathrm{T}$-cell antigen receptor (TCR) agonists, suggesting a potential role of p66ShcA in lymphocyte homeostasis [19]. The p66ShcA ${ }^{-1-}$ mice also develop a lupus-like autoimmune disease, which implies a possible key role of p66ShcA in regulating immunologic tolerance and the development of systemic autoimmunity. However, there has been no definite evidence of a role for Shc proteins in DC maturation, cytokine production or the expression of co-stimulatory molecules triggered by LPS.

In the present study, we briefly address the role of Shc proteins in the maturation process triggered by LPS in rat bone marrow-derived DCs, and we evaluate their contribution in the context of LPS-induced TLR4 signaling.

\section{Results \\ Transient activation and sustained induction of Shc proteins}

To test whether LPS can activate Shc, bone marrowderived DCs were treated with $0.5 \mu \mathrm{g} / \mathrm{ml}$ LPS for $48 \mathrm{hrs}$. When dendritic cells obtained from LEW rats were cultured with rGM-CSF and rIL-4, p46Shc and p52Shc were expressed in immature DCs, but the inducible form of p66Shc was not observed, as shown by Western blot analysis (Figure 1A). At $48 \mathrm{~h}$ after the addition of endotoxin, the expression of p66Shc was induced, and the accumulation of p52Shc was also increased. However, no significant change was observed in p46Shc accumulation.

Shc phosphorylation mechanisms were also examined in the LPS-induced maturation of DCs. Figure 1B shows that p52Shc protein was transiently phosphorylated on tyrosine residues $239 / 240$ but not 317 . We further evaluated whether the addition of anti-Shc mAb could block the phosphorylation events of Shc in DCs exposed to LPS. As shown in Figure 1B, the LPS-induced transient phosphorylation of Shc protein in YY239/240 was down-regulated by 24-h preincubation of anti-Shc monoclonal antibody. This result suggests that the antiShc mAb we used could act as a blocking antibody for Shc neutralization. Moreover, we evaluated the role of non-receptor Src-family kinases (SFKs) in Shc phosphorylation by using a small molecule Src-family kinase inhibitor SU6656 while DCs was exposed to LPS. The inhibition of Shc phosphorylation by SU6656 indicating the involvement of SFKs activity in LPS-triggered Shc signaling events. Therefore, tyrosine phosphorylation on positions 239/240 of Shc is Src-dependent.

\section{Effect of Shc silencing on the response of DCs to LPS exposure}

To evaluate the role of Shc proteins in DC cytokine production, a neutralizing anti-Shc mAb was added to immature DCs, which concomitantly treated with LPS for $24 \mathrm{~h}$. As shown in Figure 2, incubation of DCs with anti-Shc mAb but not with control mouse IgG in the presence of LPS for 24 h elicited a lower level of IL-6 secretion (Figure 2A). To determine whether the endogenous Shc directly involved in LPS-induced IL-6 production, the Shc siRNA was used. Shc siRNA dosedependently inhibited the expression of Shc mRNA expression (Figure 2B). Figure $2 \mathrm{C}$ also shows that LPSinduced IL- 6 production was inhibited by Shc siRNA. Decreased secretion of IL- 6 by 2.5 -fold in DCs stimulated with LPS and concomitantly with transfected Shc siRNA (Figure 2C).

In addition to IL-6, LPS induces DC production of various cytokines, including IL-10 and IL-12. Therefore, the homeostasis of inflammation versus immune suppression controlled by DCs via the balance of produced cytokines would be crucial, so we determined the role of Shc in IL-10 and IL-12 secretion. DCs were treated with LPS $(0.5 \mu \mathrm{g} / \mathrm{ml})$ for $24 \mathrm{~h}$ in the presence of transfected Shc siRNA (50 nM) or NEG. siRNA (50 nM), and the secretion of IL-10 and IL-12p70 in the supernatant were measured by ELISA. The mRNA expression of IL-10 and IL-12p40 was also determined by quantitative RTPCR. We found that LPS induced substantial secretion 
A

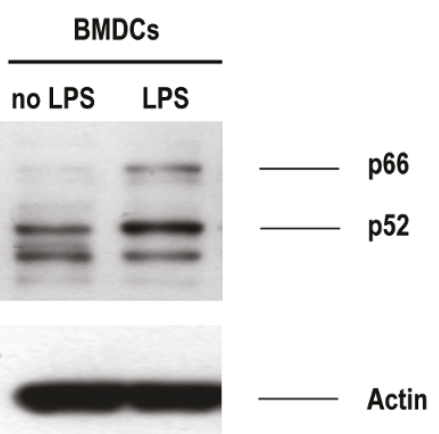

B

pTyr239/240-Shc

p52Shc

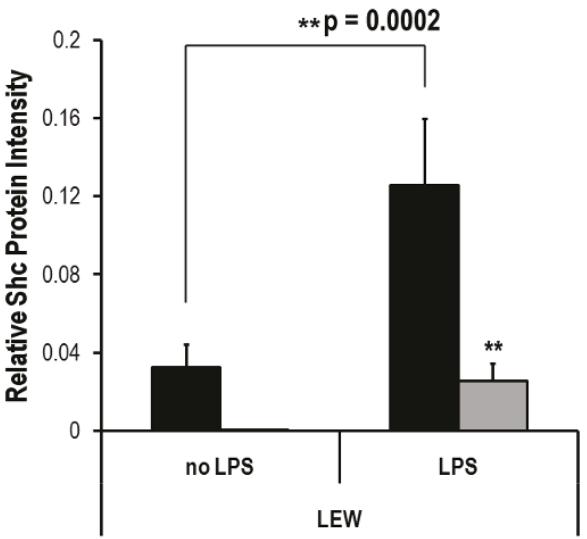

p52 SHC

口p66 SHC

pTyr317-Shc

no LPS

LPS+anti-Shc
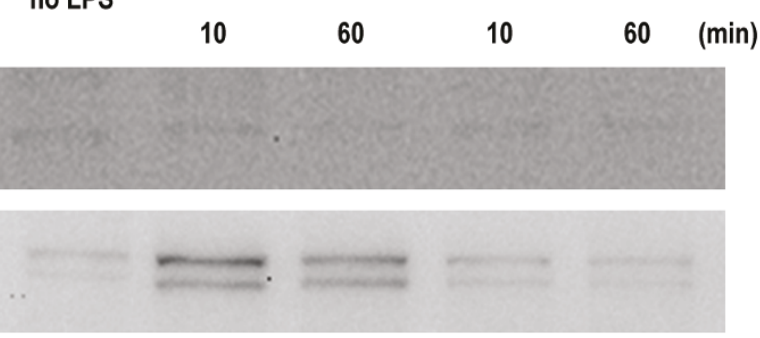
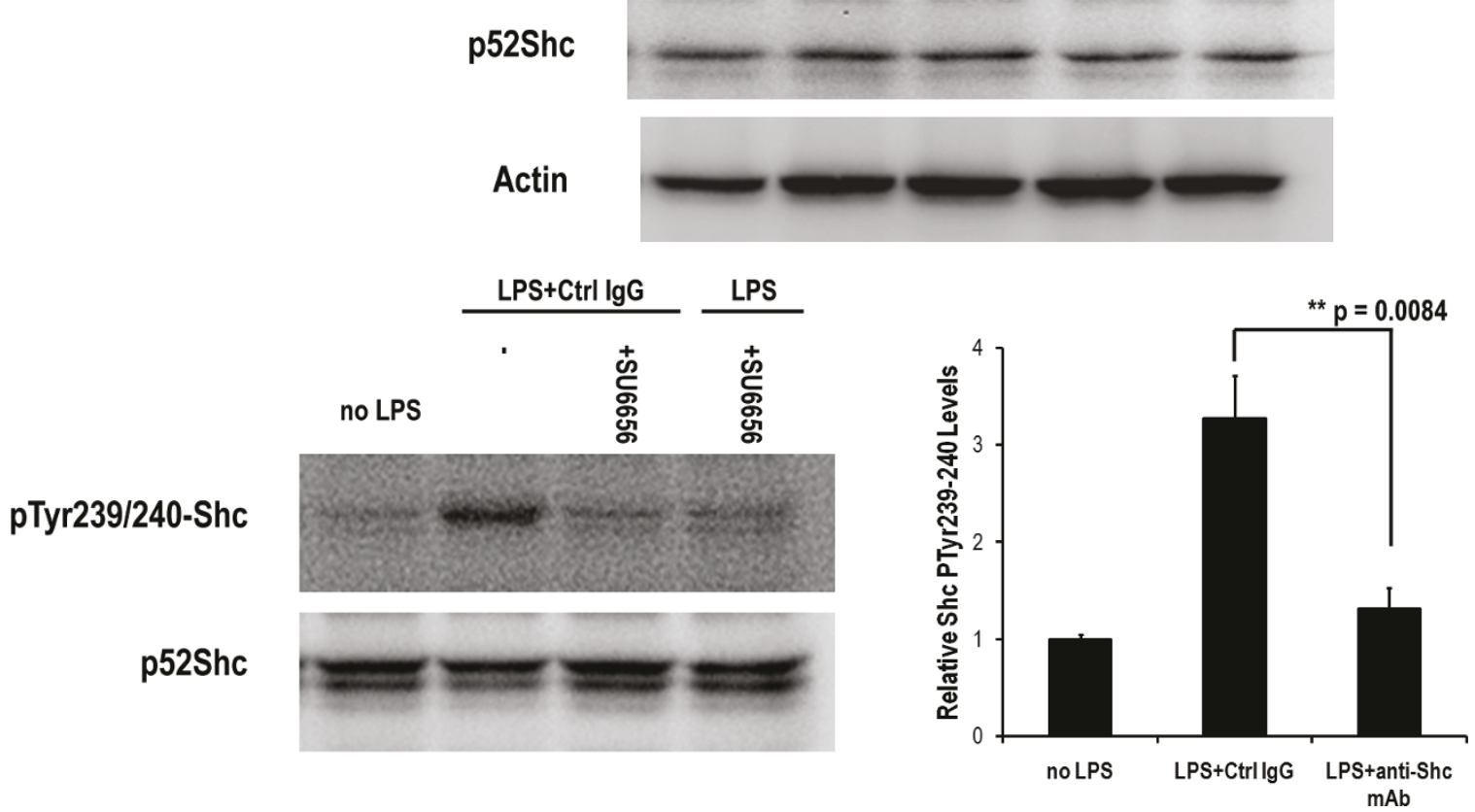

Figure 1 LPS stimulates Shc protein accumulation and activation in bone marrow-derived DCs. Purified DCs were stimulated with LPS (0.5 $\mu \mathrm{g} / \mathrm{ml})$, and Shc protein accumulation was measured at $48 \mathrm{~h}$ (A). For Shc activation, DCs were treated with LPS $(0.5 \mu \mathrm{g} / \mathrm{ml})$ with mouse control lgG or anti-Shc mAb $(2 \mu \mathrm{g} / \mathrm{ml})$ for 10 or $30 \mathrm{~min}$, or with LPS $(0.5 \mu \mathrm{g} / \mathrm{ml})$ in the presence of $10 \mathrm{nM} \mathrm{SU6656} \mathrm{and/or} \mathrm{mouse} \mathrm{control} \mathrm{lgG}(2 \mu \mathrm{g} / \mathrm{ml})$ for $10 \mathrm{~min}$, as indicated (B). Cell lysate $(30 \mu \mathrm{g})$ from each sample was subjected to Western blot with anti-Shc, anti-phosphotyrosine 317-Shc (pTyr317-Shc) and anti-phosphotyrosine 239/240-Shc (pTyr239/240-Shc) mAb. The bound antibodies were detected by the ECL system. The significant change of pTyr239/240-Shc was observed and the statistics at 10-min was presented. The results are representative of three independent experiments. $\left(n=3\right.$; $\left.{ }^{* *}, P<0.01\right)$ 

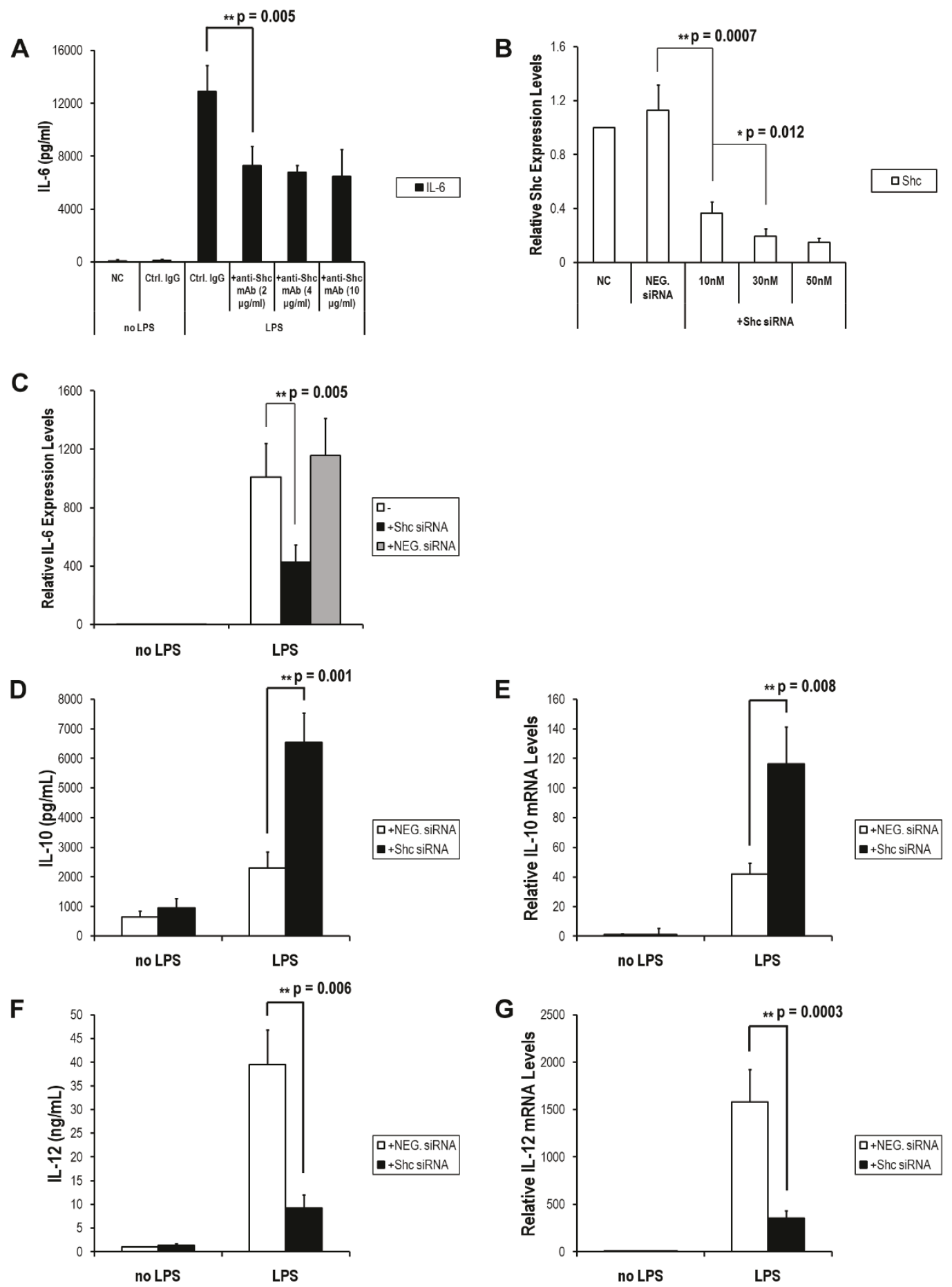

Figure 2 Effects of Shc blockade on the secretion and mRNA expression of IL-6, IL-10 and IL-12 in DC maturation. Purified DCs were cultured with or without anti-mouse Shc mAb at various concentrations, as indicated, and stimulated with LPS (0.5 $\mu \mathrm{g} / \mathrm{ml})$ for $24 \mathrm{~h}$ and the IL-6 secretion was measured in triplicate supernatants by ELISA (A). DCs were transfected with Shc siRNA or negative control siRNA (NEG. siRNA) as described in the Materials and Methods. Quantitation of the RNAi efficiency for Shc silencing was analyzed by quantitative PCR to confirm the blockade effect of the Shc siRNA $24 \mathrm{~h}$ after transfection (B). The relative expression levels of IL-6 mRNA were measured in BMDCs stimulated by LPS in the absence or presence of Shc siRNA $(50 \mathrm{nM})$, or NEG. siRNA $(50 \mathrm{nM})$ for $24 \mathrm{~h}$. Cells were collected, RNA was isolated, and IL-6 mRNA was determined by RT-PCR (C). Moreover, the amount of IL-10 and IL-12 were also determined by ELISA and RT-PCR. The data show enhanced secretion and mRNA level of IL-10 (D, E) and reduced production of IL-12 $(F, G)$ from DCs in the presence of Shc siRNA. Each column represents the mean \pm SE of the three independent experiments $\left(n=3 ;{ }^{*}, P<0.05 ;{ }^{*}, P<0.01\right)$. 
of IL-10 and increased expression of IL-10 mRNA by DCs. Notably, the LPS-induced production of IL-10 was significantly enhanced in the presence of Shc siRNA (Figure 2D and 2E). In contrast, cultured DCs showed vigorous secretion of IL-12p70 and mRNA expression of IL-12p40 in response to LPS treatment, while the IL-12 levels were significantly suppressed by Shc siRNA (Figure $2 \mathrm{~F}$ and $2 \mathrm{G}$ ). Therefore, Shc-mediated signaling appears to be crucial for regulation of IL-10 and IL-12 production by DCs upon LPS stimulation.

\section{Enhanced expression of T cell co-stimulatory molecule CD80 on Shc-silenced DCs in response to LPS exposure}

DCs undergo a maturation process while the host detects a microbial infection, which enhances their ability to process and present exogenous antigen. Optimal antigen presentation of DCs requires the expression of specific membrane protein markers. Maturation of DCs is associated with profound changes in the surface phenotype of protein markers, including an up-regulated expression of co-stimulatory and MHC class II molecules [20,21]. During maturation, the co-stimulatory molecules (CD80, CD86) are required for $\mathrm{T}$ cell priming and produce cytokines that direct $\mathrm{T}$ cell differentiation, whereas processed antigen fragments are present to the surface using MHC-II molecules. In order to evaluate the effects of Shc blockade on co-stimulatory and MHC class II molecules during DC maturation, the immature DCs were transfected with Shc siRNA prior to LPS treatment and the surface protein expression was measured by flow cytometry. CD80, CD86 and MHC-II protein levels were increased significantly in DCs expose to LPS for $24 \mathrm{~h}$. Moreover, we found that DCs treated with LPS for $24 \mathrm{~h}$ with transfected Shc siRNA display enhanced level of CD80 ( $\mathrm{P}<0.05)$, but not CD86 or MHC-II (Figure 3), indicating that CD80 was selectively enhanced during LPS-triggered maturation with Shc blockade.

\section{LPS-induced STAT3 phosphorylation and nuclear} translocation are highly enhanced by Shc silencing in DCs We further investigated STAT3 phosphorylation in LPSinduced DCs with or without Shc blockade. As shown in Figure 4, STAT3 was highly phosphorylated at $6 \mathrm{~h}$ in LPS-stimulated DCs, and its phosphorylation was almost down to basal after $24 \mathrm{~h}$. The phosphorylation level of STAT3 was significantly enhanced in DCs transfected with Shc siRNA but not NEG. siRNA. Moreover, the involvement of LPS-induced IL-10 secretion was also examined by using IL-10 neutralizing antibody (Figure 5). Our data suggests the LPS-induced STAT3 activation and the enhanced STAT3 activation via Shc blockade are IL-10 dependent. Furthermore, SU6656 totally abolished the induced STAT3 phosphorylation indicating the involvement of SFKs activity in STAT3 activation of LPS-stimulated DCs.

The nuclear translocation of phospho-STAT3 was measured. As shown in Figure 6, Shc blockade promotes nuclear localization of phospho-STAT3. STAT3 was detected mainly in the cytosolic fraction of DCs with a variety of treatment conditions. The relative phosphorylation levels of STAT3 in both cytosolic and nuclear fractions were increased by LPS treatment for $6 \mathrm{~h}$. Moreover, the relative phosphorylation level of STAT3 in the nuclear fraction (N) but not in the cytosol (C) was highly enhanced by LPS treatment with Shc blockade. This result indicates that more STAT3 phosphorylated on Tyr-705 is generated into the nucleus in LPSinduced DCs with Shc blockade. The activation of STAT3 by LPS with or without Shc blockade was totally abolished by SU6656 both in N and C fractions.

\section{Discussion}

We show that expression of Shc proteins was induced by LPS and that Shc blockade inhibited LPS-primed phosphorylation of p52Shc on tyrosine residues. IL-6 and IL-12 production triggered by LPS were suppressed by Shc blockade. Moreover, the expressions of costimulatory molecule CD80 and of IL-10 were enhanced while silencing endogenous Shc on LPS-induced DCs. However, the LPS-induced IL-10 production would be totally abolished in the presence of SU6656, implying that the signals transducing via adaptor protein Shc negatively regulate the IL-10 expression and underscoring the complexity of the role of SFKs.

Adaptive immunity evolved from an ancient innate defense mechanism common to most microorganisms. Therefore, it is not surprising that sensing and signal transduction in innate immunity is the starting point of the specific immune response. APCs, such as macrophages/monocytes and DCs, activated by various antigens of bacteria [22,23] and other microorganisms [24-26] through Toll-like receptors (TLRs), could be central to this process that integrates innate information and conveys it to lymphocytes [27]. Adaptor proteins differ from other signaling molecules in their lack of inherent catalytic activity, but they are primarily composed of modular domains that bind and act as scaffolds for the organization of macromolecular complexes, and they recruit other signaling proteins for correct localization. They play important roles in the integration and propagation of signals that lead to lymphocyte development and homeostasis [28]. Therefore, the mechanisms underlying TLR-mediated activation of APCs have been extensively analyzed, but the downstream signaling pathways governing the release of inflammatory mediators in response to LPS are yet to be understood. 


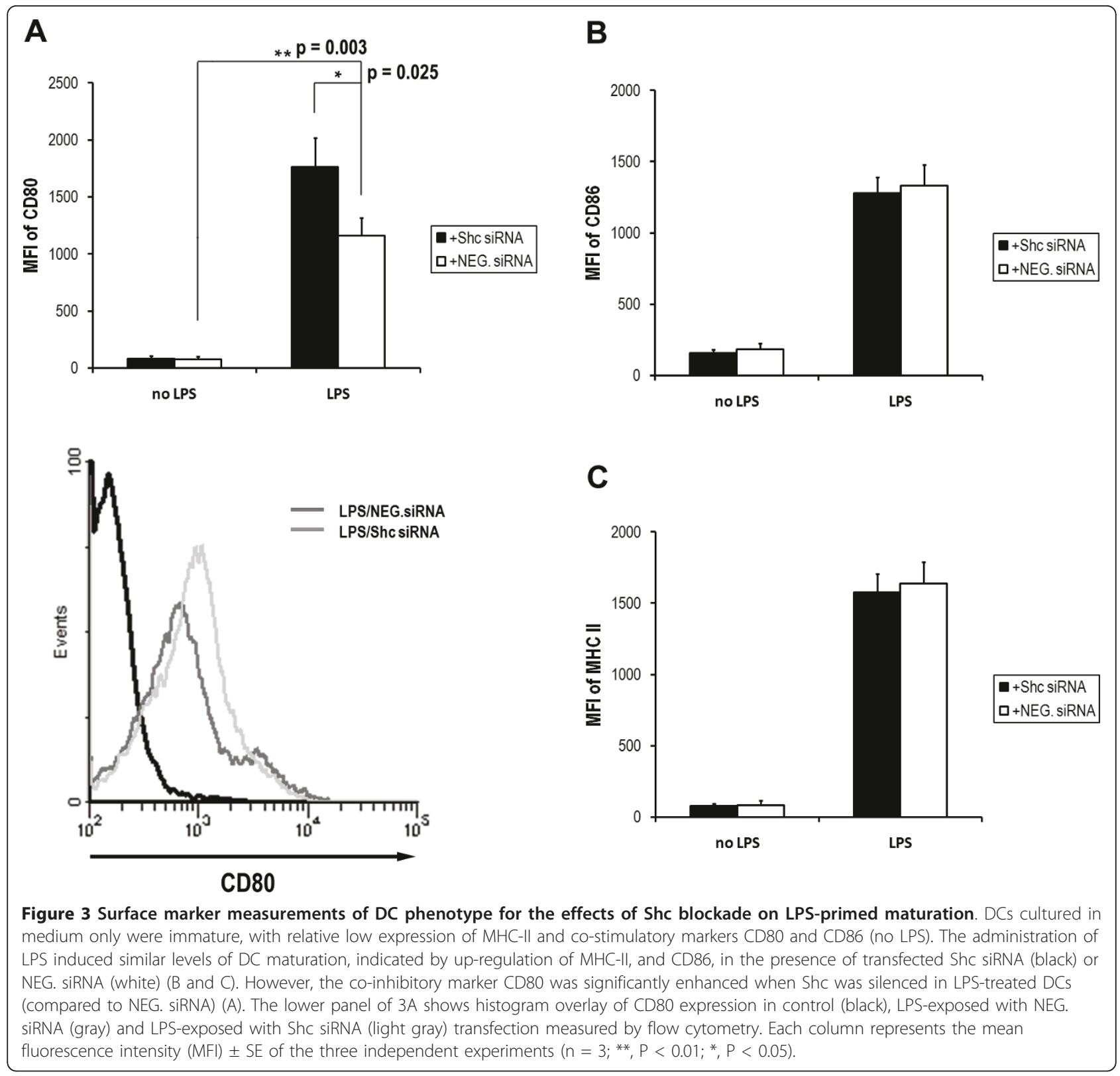

The LPS-stimulated secretion of large amounts of proinflammatory cytokines requires the recruitment of intracellular adaptor molecules. For example, myeloid differentiation primary response gene 88 (MyD88) is recruited to activate the NF- $\kappa \mathrm{B}$ and MAPK pathways via TNF receptor-associated factor 6 (TRAF6) and the IL-1 receptor-associated kinases (IRAKs) [29], and a second pair of Toll-IL-1-resistance (TIR) domain-containing adaptors inducing interferon-beta (TRIF) and TRIFrelated adaptor molecule (TRAM) induce signaling via TRAF3 and the interferon regulatory factors (IRFs) to activate the interferon response [30]. However, there is growing evidence that other adaptor molecules could also make important contributions to the inflammatory responses. The role of p52Shc in transducing signals for proliferative responses to TCR agonists has been reported [31] and the negative regulation in T-cell and B-cell activation via p66Shc using $\mathrm{p}^{66^{-1-}}$ mice has also been illustrated [19].

The involvement of Shc members in signaling pathways have been well characterized $[17,32,33]$. The $\mathrm{CH} 1$ domain has three tyrosine residues, 239/240 and 317 in p52Shc, which upon phosphorylation interact with Grb2 and have been proposed to be differentially coupled to Fos-dependent proliferation signaling (Y317) and to Myc-dependent survival pathways (YY239/240) in B-cell 

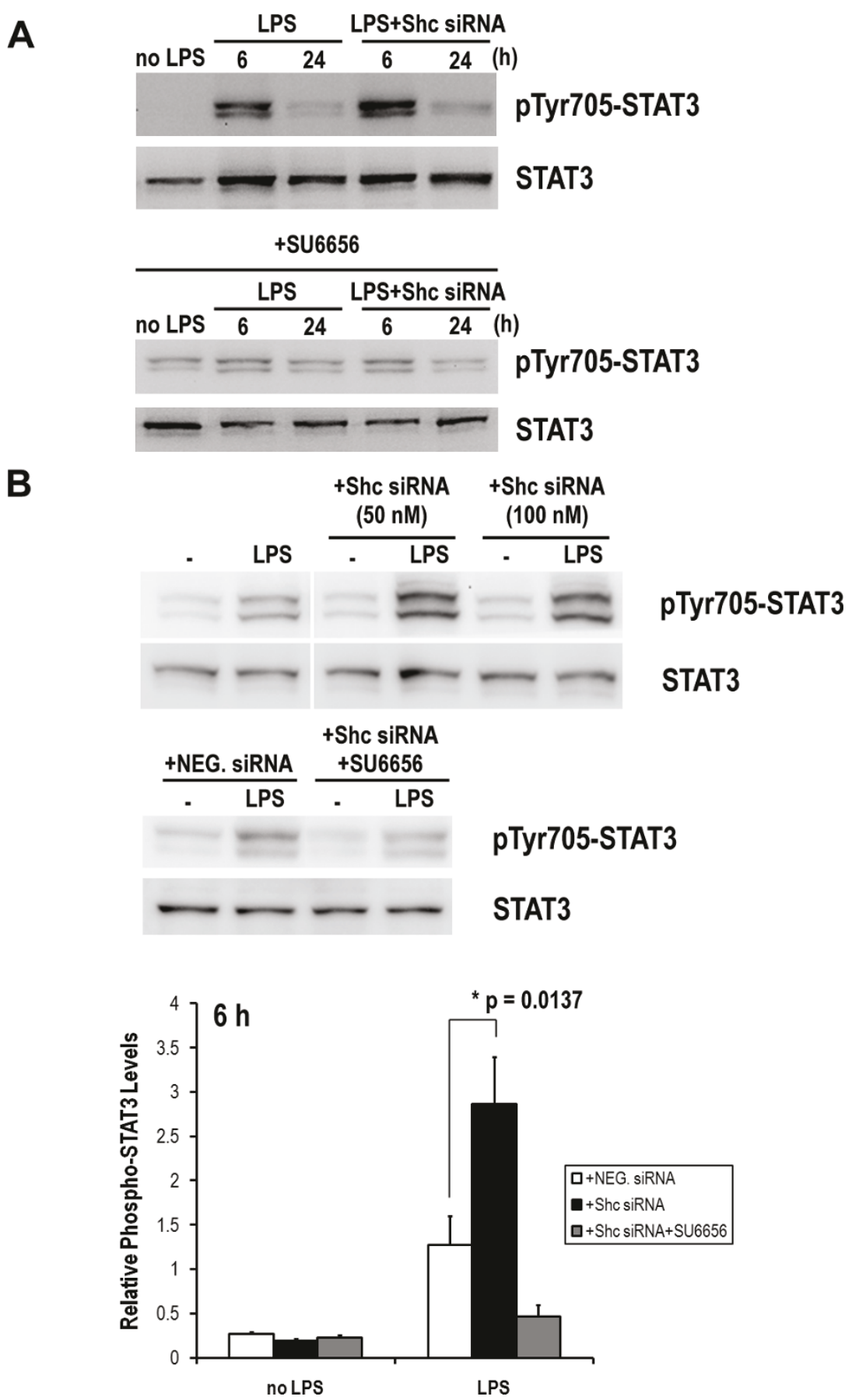

Figure 4 STAT3 phosphorylation activated by LPS is significantly enhanced by Shc blockade. DCs from Lewis rats were stimulated with LPS $(0.5 \mathrm{\mu g} / \mathrm{ml})$ in the presence of Shc siRNA $(50 \mathrm{nM}, 100 \mathrm{nM}$ ) or NEG. siRNA. Cells were harvested and Western blot analysis was performed for phospho-STAT3 at Tyr-705 and for total STAT3. Additional treatment of 10 nM SU6656 indicated total inhibition of STAT3 phosphorylation at Tyr705. Each column represents the mean \pm SE of the three independent experiments $\left(n=3 ;{ }^{*}, P<0.05\right)$.

[32] and T-cell activation [34]. In the present study, we provide new insight into the role of Shc in the maturation process triggered by LPS in DC, and we evaluate their contribution in the context of LPS-induced TLR4 signaling. The proximal signaling events occurring through Shc in association with LPS-induced maturation of DC were first defined. Our study was based on the primary observation in accumulation of Shc proteins after a 48-h LPS treatment. Strong suppression of IL-6 and IL12 production by Shc siRNA as well as anti-Shc mAb in 


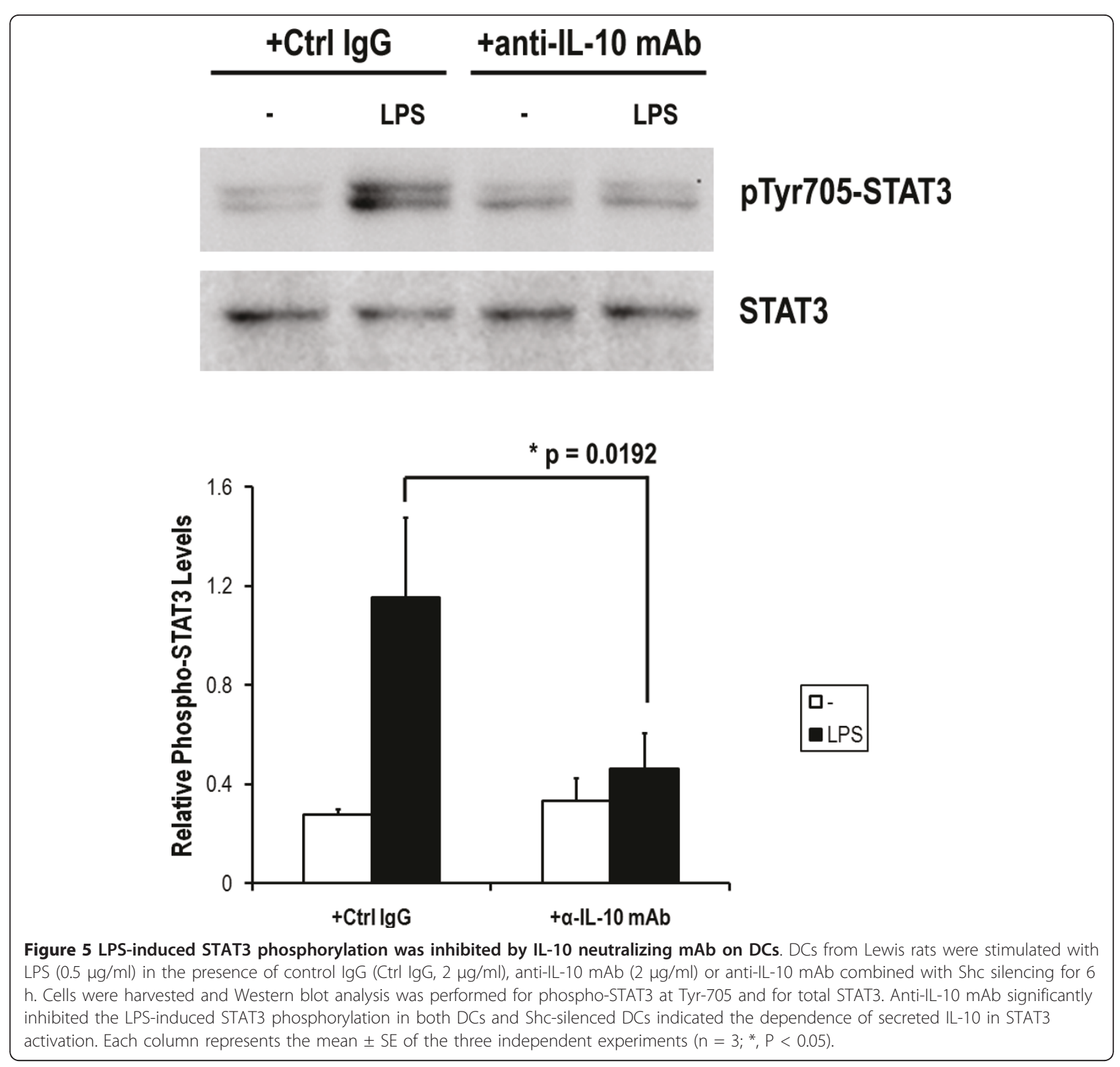

DC suggests the role of Shc signaling in promoting the pro-inflammatory properties of LPS-stimulated DC. However, the Shc-silenced DC stimulated with LPS also exhibited increased levels of costimulatory molecules and MHC II antigen stimulation. This indicates that the tolerogenic state of LPS-primed DC under Shc silencing could be determined by unravel factors through IL-10, a cytokine which has also been found in contributing to hCG-induced tolerogenic phenotype of DC [35]. Recently, the role of activated STAT3 as the transcription factor induced by IL-10 in tolerogenic DC was investigated [36,37]. DCs expressing activated STAT3 produce less IL-12p40 by inhibiting the recruitment but not activation of NF- $\kappa \mathrm{B}$ subunits [38]. In this study, we found that Shc blockade significantly enhances STAT3 phosphorylation on tyrosine residue-705 and increases the nuclear localization of phospho-STAT3 in a Src-dependent manner. This finding establishes a novel signaling event for STAT3 and associates its activation with a negative regulatory role for Shc in tolerogenic properties of DC. Furthermore, it has been found that the STAT3 pathway is required for IL-10-mediated down-regulation of inflammatory genes in monocytes by LPS $[39,40]$, and STAT3 phosphorylation is likely due to LPS-induced IL10 secretion in DC, while no phospho-STAT3 was observed in LPS-stimulated IL-10 ${ }^{-1-}$ BMDCs [38]. Our results are consistent with these studies in that LPSinduced STAT3 phosphorylation was blocked by IL-10 


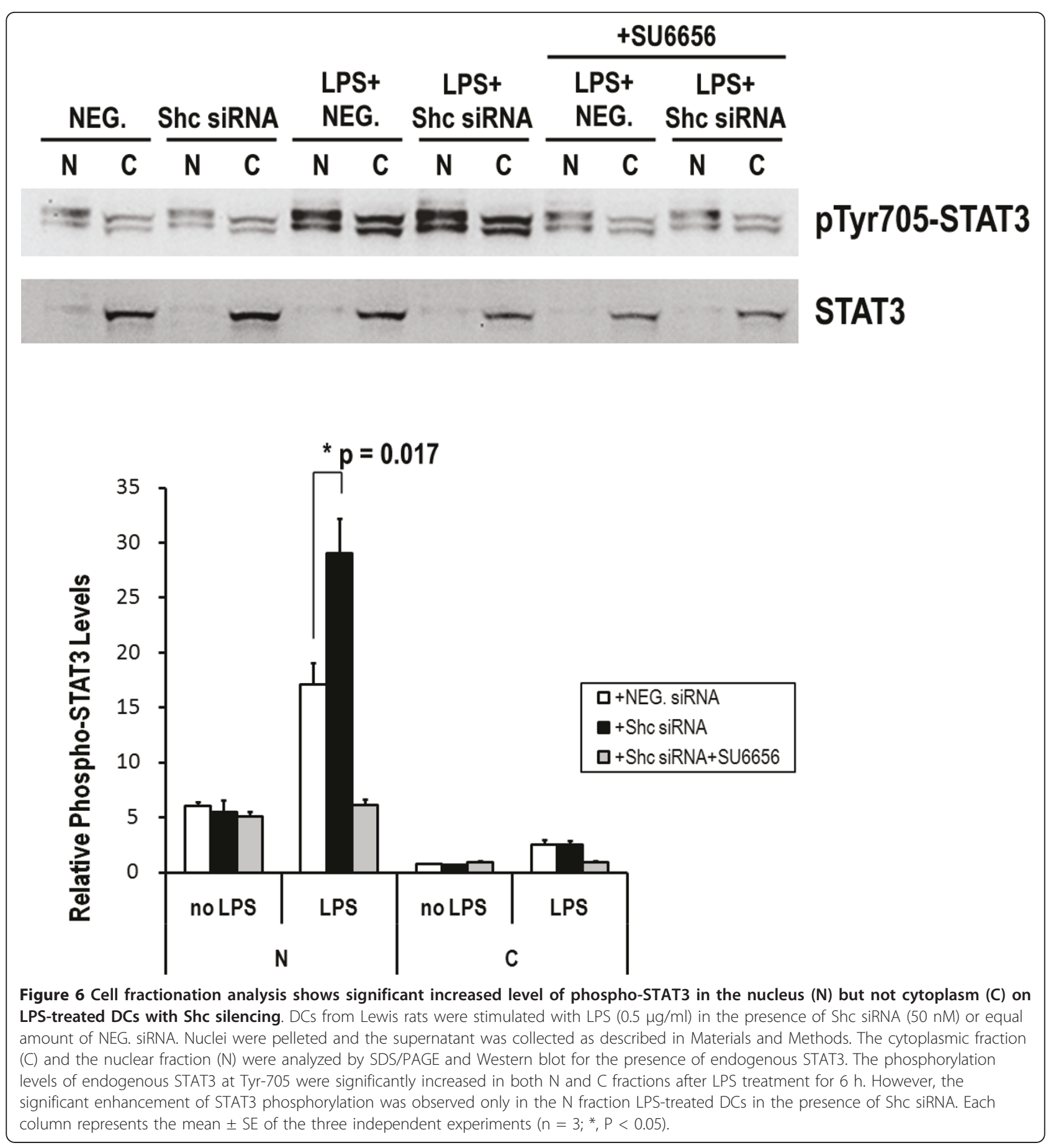

neutralization. LPS-induced $\mathrm{I} \kappa \mathrm{B}$ degradation was not affected by Shc blockade and Src inhibitor SU6656 (data not shown). In conjunction with a similar activation profile for Erk1/2 and p38 signaling events, these data suggest that LPS-triggered Src-Shc signaling events are not involved directly in the signaling cascade which has been reported in causing increased IL-12p40 gene expression via other adaptor proteins through MAPK and NF- $\kappa \mathrm{B}$ pathways $[39,41]$. The TLR-stimulated signaling cascades via activation of transcription factors NF- $\kappa \mathrm{B}, \mathrm{AP}-1$ and IRF3, results in the expression of "innate" genes involved in pro-inflammatory properties of mature DC were not determined by Src-family kinase activity. However, the pro-inflammatory properties of mature DC were directed to Th2 phenotype and determined by Src-mediated STAT3 activation while Shc was blocked. 
Furthermore, Shc proteins are key components of the pathways that activate downstream signaling molecules of growth factor receptors, extracellular matrix proteins and mechanical forces $[16,17,42]$, and have been revealed a role in inflammatory signaling with increased ECM binding [43]. A recent study also showed that LPS-induced DC is enriched in MHC-II antigen and intercellular adhesion molecule 1 (ICAM-1) in the vesicular compartment called exosomes [44]. The enriched MHC-II and ICAM-1 are required for mature DC to prime naïve $\mathrm{T}$ cells representing the immunogenic activity of LPS-treated DCs. We briefly found that co-immunoprecipitation of p52Shc and ICAM-1 in LPS-treated DCs (data not shown) implying the involvement of adhesion molecules via Shc in modulating the immunogenic and tolerogenic properties of mature DC. However, the detailed mechanisms for the interplay of extracellular matrix, adaptor proteins as well as specific SFKs are critical in the regulation of DC-mediated immune response and require further investigation.

\section{Conclusions}

In summary, we propose a model where the LPS-primed mature DC produce high level of IL-10 as well as nuclear transactivation of Src-mediated phospho-STAT3 while Shc-mediated signaling is blocked. Our findings suggest that the balance of inflammatory versus antiinflammatory cytokines generated by DCs upon TLR stimulation could be critically determined by Shc, which may act like a switch in response to subsequently acquired immunity.

\section{Methods}

Rat

Four-week-old male Lewis (LEW; RT1 ${ }^{\mathrm{l}}$ ) rats were obtained from the National Laboratory Animal Breeding and Research Center (Taipei, Taiwan). All animals were maintained in specific pathogen-free animal facilities with water and commercial rat food provided ad libitum. Our experimental design was reviewed and approved by our institutional experimental animal committee.

Preparation, transfection and treatment of bone marrowderived dendritic cells

Thigh bones obtained from LEW rats were cleaned of muscle tissue and placed in sterile Petri dishes containing complete medium [RPMI-1640 with $10 \%$ fetal bovine serum (FBS), penicillin/streptomycin $100 \mathrm{U} / \mathrm{ml}$ and 100 $\mathrm{mg} / \mathrm{ml}$ and $2 \mathrm{mM} \mathrm{L}$-glutamine], following the procedures described elsewhere [45]. On day 8, the non-adherent cells were aspirated for DCs culture. Briefly, the wells were washed once with medium, and the cells were pooled. DCs $\left(1 \times 10^{6}\right.$ cells $)$ were placed in a new six-well plate and cultured in fresh DC culture medium (DCM) containing $50 \mathrm{ng} / \mathrm{ml}$ recombinant granulocyte-monocyte colony-stimulating factor (rGM-CSF), $50 \mathrm{ng} / \mathrm{ml} \mathrm{rIL-4}$ (CytoLab, Ltd, Rehovot, Israel) and endotoxin (lipopolysaccharide, LPS; $0.5 \mu \mathrm{g} / \mathrm{ml}$; Sigma, St Louis, MO, USA) with the addition of anti-Shc monoclonal antibody (mAb, $2 \mu \mathrm{g} / \mathrm{ml}$ ) (Santa Cruz Biotechnology, Santa Cruz, CA, USA) and mouse IgG $(2 \mu \mathrm{g} / \mathrm{ml})$ (Santa Cruz Biotechnology) for $24 \mathrm{~h}$. For transient transfection experiments, DCs were transfected with $50 \mathrm{nM}$ of Shc siRNA or Silencer $^{\circledR}$ negative control siRNA (NEG. siRNA) (Ambion Inc., Austin, TX, USA) using GenMute ${ }^{\mathrm{TM}}$ (SignaGen Laboratories, Gaithersburg, MD, USA) for $24 \mathrm{~h}$.

In some experiments, DCs $\left(1 \times 10^{6}\right.$ cells $)$ were treated with a selective Src family kinase inhibitor SU6656 (10 nM) (Sigma, St. Louis, MO, USA) in the presence of LPS stimulation with or without Shc blockade.

\section{Cytokine detection in ELISA}

After 24-h LPS stimulation of DCs with or without Shc blockade, supernatants were harvested and assayed for cytokine production using commercially available ELISA specifically for the rat IL-12p70 (eBioscience Inc., San Diego, CA, USA), IL-10 (R\&D Systems Inc., Minneapolis, MN, USA) and IL-6 (R\&D Systems Inc.) according to the manufacturer's instructions.

\section{RNA isolation and real-time PCR}

Total RNA was isolated from DCs using the RNeasy kit from Qiagen (Valencia, CA, USA), according to the manufacturer's instructions. Reverse transcripts were performed using the First-Strand cDNA synthesis kit (Promega, Madison, WI, USA) following the manufacturer's recommendations. Total RNA $(1 \mu \mathrm{g})$ was transcribed to cDNA in a $20-\mu$ reaction volume. The quantitative $P C R$ reaction was performed on an $A B I$ 7500 Fast Real-Time PCR System with the SDS 1.4 program and using the ABI TaqMan Fast Universal PCR master mix (Applied Biosystems, Foster, CA, USA). The primers and TaqMan MGB probes of IL-10 (Rn00563409_m1) and IL-12 (Rn00575112_m1) were obtained from Applied Biosystems, and the final concentration of primers and probes was $300 \mathrm{nM}$ and $250 \mathrm{nM}$, respectively. The cycling profile for each run was $95^{\circ} \mathrm{C}$ for 20 seconds and 40 cycles of $95^{\circ} \mathrm{C}$ for 3 seconds followed by $60^{\circ} \mathrm{C}$ for 30 seconds, using the default ramp rate. Normalization was performed by using rat GAPDH primers. Comparative real-time PCR (RT-PCR) data was analyzed in triplicate, including non-template controls. Fold increase in the expression of cytokine mRNA was calculated by using the comparative $2^{\Delta \mathrm{Ct}}$ method.

\section{Antibodies and Western blot analysis}

Cell lysates (30-40 $\mu \mathrm{g}$ ) were electrophoresed and performed Western blot analysis as previously described [46]. 
Antibodies to phospho-Shc (Tyr239/240 or Tyr317) and total Shc, phospho- and total STAT3 were all purchased from Cell Signaling (Cell Signaling Technology, Inc., Danvers, MA, USA). Antibody to Shc blocking (PG-797) was purchased from Santa Cruz Biotechnology (Santa Cruz, CA, USA). Antibody to IL-10 neutralization (ARC9102) was purchased from Invitrogen (Carlsbad, CA, USA). Antibody to $\alpha$-actin was obtained from Santa Cruz Biotechnology. Appropriate HRP-conjugated, secondary antibodies were used to visualize the specific bands. Detection was performed with the Immobilon Western HRP Substrate (Millipore, Billerica, MA, USA), and relevant bands were quantified by densitometry using UVI photo version 99 and TotalLab software version 1.00 (Nonlinear USA Inc., Durham, NC, USA).

\section{Nuclear and cytoplasmic protein extraction}

DCs with different treatment conditions were washed twice in PBS, harvested, and pelleted by centrifugation at $500 \times \mathrm{g}$ for 3 min. CERI (NE-PERTM, Thermo Fisher Scientific Inc., Rockford, IL, USA) was added to each sample following the supplier's guideline $(200 \mu \mathrm{l}$ of CERI per $20-\mu \mathrm{l}$ cell volume). Briefly, cells were resuspended by vortexing for 15 seconds and then incubated on ice for $10 \mathrm{~min}$. CERII was then added to each sample $(11 \mu$ l of CERII solution for every $20-\mu$ l cell volume) followed by repeated vortexing and centrifugation at $13,000 \times \mathrm{g}$ for $5 \mathrm{~min}$. The supernatant (cytoplasmic extract) was immediately stored on ice. The insoluble nuclear pellet was resuspended in $100 \mu \mathrm{l}$ of NER (NE-PERTM, Thermo Fisher Scientific Inc.) per 20- $\mu \mathrm{l}$ cell volume and incubated on ice for $40 \mathrm{~min}$ with intermittent brief vortexing. The samples were centrifuged at $13,000 \times \mathrm{g}$ for $10 \mathrm{~min}$, and the supernatant (nuclear extract) was stored on ice. After measuring the total volumes of each fraction, their protein concentrations were measured using the Thermo protein assay reagent. All cytoplasmic and nuclear protein fractions were stored at $-80^{\circ} \mathrm{C}$ and ready for further Western blot analysis.

\section{FACS analysis}

DCs $\left(1 \times 10^{6}\right.$ cells $\left./ \mathrm{ml}\right)$ were harvested in RPMI complete medium and were washed twice with PBS containing $0.1 \%$ sodium azide plus $2 \%$ heat-inactivated FBS (wash buffer). Cells were incubated with various $\mathrm{mAb}$ [fluorescein isothiocyanate (FITC)-anti-CD80, FITC-anti-CD86, FITC-anti-MHC class II] at $4^{\circ} \mathrm{C}$ for $40 \mathrm{~min}$. All the fluorescein-conjugated $\mathrm{mAb}$ were obtained from BD Pharmingen (Franklin Lakes, NJ, USA) or eBioscience, and isotype-matched Ab used as controls were purchased from Santa Cruz Biotechnology. After extensive washing, the cells were fixed in $4 \%$ paraformaldehyde. Stained DC, gated according to forward- and side-scatter characteristics, were analyzed on Epics ${ }^{\circledR}$ ALTRA $^{\mathrm{TM}}$ flow cytometer (Beckman
Coulter, Inc., Fullerton, CA, USA) using EXPO32 software. Fluorescence data were expressed as percentage of positive cells compared to immature DCs.

\section{Statistical analysis}

The statistical significance of the data was calculated using Student's $t$-test. A value of $P<0.05$ was considered to indicate statistical significance.

\section{List of abbreviations}

APC: antigen presenting cell; BMDCs: bone marrow-derived dendritic cells; DCs: dendritic cells; ECM: 1/2; GAPDH: glyceraldehyde 3-phosphate dehydrogenase; HRP: horse radish peroxidase; IFNs: interferons; ILextracellular matrix; ELISA: enzyme-linked immunosorbent assay; ERK 1/2: extracellular signal-regulated kinase 10: interleukin-10; IL-12: interleukin-12; IL6: interleukin-6; LPS: lipopolysaccharide; mAb: monoclonal antibody; NF-KB: nuclear factor-kappa B; rGM-CSF: recombinant granulocyte-monocyte colonystimulating factor; RT-PCR: real-time polymerase-chain reaction; rll-4: recombinant interleukin-4; SFKs: Src-family kinases; Shc: Src homology 2 domain containing; siRNA: small interfering RNA; STAT3: signal transducer and activator of transcription 3; TLRs: toll-like receptors.

\section{Acknowledgements}

We gratefully acknowledge Dr. Yur-Ren Kuo (Department of Plastic and Reconstructive Surgery, Chang Gung Memorial Hospital, Kaohsiung, Taiwan) for helpful discussions and technical supports regarding the research. This work was supported in part by grants from the National Science Council (NSC98-2314-B-182A-058-MY3 to C-LC; NSC98-2314-B-182A-050-MY2 to Y-FC; NSC98-2320-B-182-029-MY3 to TN) and the Chang Gung Memorial Hospital (CMRPG871341; CMRPG891061 to C-LC; CMRPG890721 to Y-FC;

CMRPD880011 to TN; CMRPG881232 to C-CW; CMRPG870902 to K-WC; CMRPG881081 AND CMRPG881082 to K-DC) of Taiwan.

\section{Disclosures}

The authors have no financial conflicts of interest.

\section{Author details}

${ }^{1}$ Center for Translational Research in Biomedical Sciences, Liver

Transplantation Program, Departments of Surgery, Kaohsiung Chang Gung Memorial Hospital and Chang Gung University College of Medicine, Kaohsiung, Taiwan. ${ }^{2}$ Department of Chemistry, National Cheng Kung University, Tainan, Taiwan. ${ }^{3}$ wao Hospital, Yufuin, Japan. ${ }^{4}$ Graduate Institute of Clinical Medical Sciences, Chang Gung University College of Medicine, Kaohsiung, Taiwan. ${ }^{5}$ Diagnostic Radiology, Chang Gung Memorial HospitalKaohsiung Medical Center, Kaohsiung, Taiwan. ' ${ }^{6}$ Hepato-gastroenterology, Chang Gung Memorial Hospital-Kaohsiung Medical Center, Kaohsiung, Taiwan.

\section{Authors' contributions}

KDC designed research, performed research, analyzed data and wrote the paper; LWH designed research, performed research; SG designed research and wrote the paper; CWY performed research and analyzed data; TN contributed analytical tools and platforms; CYL performed research; $\mathrm{CHH}$ performed research; YCC performed research; CCW contributed analytical tools and platforms; YFC contributed analytical tools and platforms KWC contributed analytical tools and platforms CCL contributed analytical tools and platforms CLC designed research and wrote the paper. All authors read and approved the final manuscript.

Received: 7 December 2010 Accepted: 25 May 2011

Published: 25 May 2011

\section{References}

1. Steinman RM, Kaplan G, Witmer MD, Cohn ZA: Identification of a novel cell type in peripheral lymphoid organs of mice. V. Purification of spleen dendritic cells, new surface markers, and maintenance in vitro. J Exp Med 1979, 149:1-16. 
2. Banchereau J, Steinman RM: Dendritic cells and the control of immunity. Nature 1998, 392:245-252.

3. Lanzavecchia A, Sallusto F: Regulation of T cell immunity by dendritic cells. Cell 2001, 106:263-266.

4. Maldonado-Lopez R, Moser M: Dendritic cell subsets and the regulation of Th1/Th2 responses. Semin Immunol 2001, 13:275-282.

5. Moser M, Murphy KM: Dendritic cell regulation of TH1-TH2 development. Nat Immunol 2000, 1:199-205.

6. Chakraborty A, Li L, Chakraborty NG, Mukherji B: Stimulatory and inhibitory maturation of human macrophage-derived dendritic cells. Pathobiol 1999, 67:282-286.

7. Chakraborty A, Li L, Chakraborty NG, Mukherji B: Stimulatory and inhibitory maturation of human myeloid dendritic cells. Clin Immunol 2000, 94:88-98.

8. Corinti S, Albanesi C, la Sala A, Pastore S, Girolomoni G: Regulatory activity of autocrine IL-10 on dendritic cell functions. J Immunol 2001, $166: 4312-4318$.

9. Willems F, Marchant A, Delville JP, Gérard C, Delvaux A, Velu T, de Boer M, Goldman M: Interleukin-10 inhibits B7 and intercellular adhesion molecule-1 expression on human monocytes. Eur J Immunol 1994, 24:1007-1009.

10. Allavena P, Piemonti L, Longoni D, Bernasconi S, Stoppacciaro A, Ruco L, Mantovani A: IL-10 prevents the generation of dendritic cells from CD14 + blood monocytes, promotes the differentiation to mature macrophages and stimulates endocytosis of FITC-dextran. Adv Exp Med Biol 1997, 417:323-327.

11. De Smedt T, Van Mechelen M, De Becker G, Urbain J, Leo O, Moser M: Effect of interleukin-10 on dendritic cell maturation and function. Eur J Immunol 1997, 27:1229-1235.

12. Battaglia M, Gregori S, Bacchetta R, Roncarolo MG: Tr1 cells: from discovery to their clinical application. Semin Immunol 2006, 18:120-127.

13. Zhou X, Schmidtke P, Zepp F, Meyer CU: Boosting interleukin-10 production: therapeutic effects and mechanisms. Curr Drug Targets Immune Endocr Metabol Disord 2005, 5:465-575.

14. Sallusto F, Lanzavecchia A: Efficient presentation of soluble antigen by cultured human dendritic cells is maintained by granulocyte/ macrophage colony-stimulating factor plus interleukin 4 and downregulated by tumor necrosis factor alpha. J Exp Med 1994 179:1109-1118.

15. Stefanova I, Corcoran ML, Horak EM, Wahl LM, Bolen JB, Horak ID: Lipopolysaccharide induces activation of CD14-associated protein tyrosine kinase p53/56lyn. J Biol Chem 1993, 268:20725-20728.

16. Ravichandran KS: Signaling via Shc family adapter proteins. Oncogene 2001, 20:6322-6330.

17. Chen KD, Li YS, Kim M, Li S, Yuan S, Chien S, Shyy JY: Mechanotransduction in response to shear stress. Roles of receptor tyrosine kinases, integrins, and Shc. J Biol Chem 1999, 274:18393-18400.

18. Kavanaugh WM, Williams LT: An alternative to $\mathrm{SH} 2$ domains for binding tyrosine-phosphorylated proteins. Science 1994, 266:1862-1865.

19. Finetti F, Pellegrini M, Ulivieri C, Savino MT, Paccagnini E, Ginanneschi C, Lanfrancone L, Pelicci PG, Baldari CT: The proapoptotic and antimitogenic protein p66SHC acts as a negative regulator of lymphocyte activation and autoimmunity. Blood 2008, 111:5017-5027.

20. Pulendran B, Kumar P, Cutler CW, Mohamadzadeh M, Van Dyke T, Banchereau J: Lipopolysaccharides from distinct pathogens induce different classes of immune responses in vivo. J Immunol 2001, 167:5067-5076.

21. Pulendran B: Variegation of the immune response with dendritic cells and pathogen recognition receptors. J Immunol 2005, 174:2457-2465.

22. Poltorak A, He X, Smirnova I, Liu MY, Van Huffel C, Du X, Birdwell D, Alejos E, Silva M, Galanos C, Freudenberg M, Ricciardi-Castagnoli P, Layton B, Beutler B: Defective LPS signaling in C3H/HeJ and C57BL/ 10ScCr mice: mutations in Tlr4 gene. Science 1998, 282:2085-2088.

23. Means TK, Golenbock DT, Fenton MJ: The biology of Toll-like receptors. Cytokine Growth Factor Rev 2000, 11:219-232.

24. Underhill DM, Ozinsky A, Hajjar AM, Stevens A, Wilson CB, Bassetti M, Aderem A: The Toll-like receptor 2 is recruited to macrophage phagosomes and discriminates between pathogens. Nature 1999, 401:811-815.

25. Brightbill HD, Libraty DH, Krutzik SR, Yang RB, Belisle JT, Bleharski JR, Maitland M, Norgard MV, Plevy SE, Smale ST, Brennan PJ, Bloom BR, Godowski PJ, Modlin RL: Host defense mechanisms triggered by microbial lipoproteins through toll-like receptors. Science 1999, 285:732-736.

26. Means TK, Wang S, Lien E, Yoshimura A, Golenbock DT, Fenton MJ: Human toll-like receptors mediate cellular activation by Mycobacterium tuberculosis. J Immunol 1999, 163:3920-3927.

27. Kapsenberg ML: Dendritic-cell control of pathogen-driven T-cell polarization. Nat Rev Immunol 2003, 3:984-993.

28. Janssen $\mathrm{E}$, Zhang W: Adaptor proteins in lymphocyte activation. Curr Opin Immunol 2003, 15:269-276.

29. Lee MS, Kim YJ: Signaling pathways downstream of pattern-recognition receptors and their cross talk. Annu Rev Biochem 2007, 76:447-480.

30. Li X, Qin J: Modulation of Toll-interleukin-1 receptor mediated signaling. J Mol Med 2005, 83:258-266.

31. Pacini S, Pellegrini M, Migliaccio E, Patrussi L, Ulivieri C, Ventura A, Carraro F, Naldini A, Lanfrancone L, Pelicci P, Baldari CT: p66SHC promotes apoptosis and antagonizes mitogenic signaling in T cells. Mol Cell Biol 2004, 24:1747-1757.

32. Salcini AE, McGlade J, Pelicci G, Nicoletti I, Pawson T, Pelicci PG: Formation of Shc-Grb2 complexes is necessary to induce neoplastic transformation by overexpression of Shc proteins. Oncogene 1994, 9:2827-2836.

33. Gotoh N, Tojo A, Shibuya M: A novel pathway from phosphorylation of tyrosine residues $239 / 240$ of Shc, contributing to suppress apoptosis by IL-3. EMBO J 1996, 15:6197-6204.

34. Patrussi L, Savino MT, Pellegrini M, Paccani SR, Migliaccio E, Plyte S, Lanfrancone $L$, Pelicci PG, Baldari $C T$ : Cooreration and selectivity of the two Grb2 binding sites of p52Shc in T-cell antigen receptor signaling to ras family GTPases and Myc-dependent survival. Oncogene 2005, 24:2218-2228.

35. Wan $H$, Versnel MA, Leijten $L M$, van Helden-Meeuwsen CG, Fekkes $D$, Leenen PJ, Khan NA, Benner R, Kiekens RC: Chorionic gonadotropin induces dendritic cells to express a tolerogenic phenotype. J Leukoc Biol 2008, 83:894-901.

36. Murray P: The primary mechanism of the IL-10-regulated antiinflammatory response is to selectively inhibit transcription. Proc Natl Acad Sci USA 2005, 102:8686-8691.

37. Murray P: Understanding and exploiting the endogenous interleukin-10/ STAT3-mediated anti-inflammatory response. Curr Opin Pharmacol 2006, 6:379-386.

38. Hoentjen F, Sartor RB, Ozaki M, Jobin C: STAT3 regulates NF-kappaB recruitment to the IL-12p40 promoter in dendritic cells. Blood 2005, 105:689-696.

39. Takeda K, Akira S: Toll-like receptors in innate immunity. Int Immunol 2005, 17:1-14.

40. Riley JK, Takeda K, Akira S, Schreiber RD: Interleukin-10 receptor signaling through the JAK-STAT pathway. Requirement for two distinct receptorderived signals for anti-inflammatory action. J Biol Chem 1999, 274:16513-16521.

41. Land WG: Innate immunity-mediated allograft rejection and strategies to prevent it. Transplant Proc 2007, 39:667-672.

42. Pelicci G, Lanfrancone L, Grignani F, McGlade J, Cavallo F, Forni G, Nicoletti I, Grignani F, Pawson T, Pelicci PG: A novel transforming protein (SHC) with an $\mathrm{SH} 2$ domain is implicated in mitogenic signal transduction. Cell 1992, 70:1165-1174.

43. Liu Y, Sweet DT, Irani-Tehrani M, Maeda N, Tzima E: Shc coordinates signals from intercellular junctions and integrins to regulate flowinduced inflammation. J Cell Biol 2008, 182:185-196.

44. Segura E, Nicco C, Lombard B, Veron P, Raposo G, Batteux F, Amigorena S, Thery C: ICAM-1 on exosomes from mature dendritic cells is critical for efficient naïve T-cell priming. Blood 2005, 106:216-223.

45. Hsu LW, Goto S, Nakano T, Lai CY, Kao YH, Lin YC, Kawamoto S, Ono K, Lord R, Goto T, Omori N, Sato S, Chiang KC, Chen SH, Jawan B, Cheng YF, Chiu KW, Chen CL: The effects of anti-histone $\mathrm{H} 1$ antibody on immune cells responsible for rejection reaction. Mol Immunol 2005, 42:1155-1164.

46. Hsu LW, Chen CL, Nakano T, Lai CY, Chiang KC, Lin YC, Kao YH, Chen SH, Goto T, Sung WC, Yang CH, Cheng YF, Jawan B, Chiu KW, Goto S: The role of a nuclear protein, histone $\mathrm{H} 1$, on signaling pathways for the maturation of dendritic cells. Clin Exp Immunol 2008, 152:576-584.

doi:10.1186/1471-2172-12-32

Cite this article as: Chen et al:: Adaptor protein Shc acts as an immuneregulator for the LPS-stimulated maturation of bone marrow-derived dendritic cells. BMC Immunology 2011 12:32. 